\title{
BUILDING AS-IS PROCESS MODELS FROM TASK DESCRIPTIONS
}

\author{
Azeem Lodhi \\ University of Magdeburg \\ Magdeburg, Germany
}

\author{
Gamal Kassem \\ University of Magdeburg \\ Magdeburg, Germany \\ Gunter Saake \\ University of Magdeburg \\ Magdeburg, Germany \\ firstname.lastname@ovgu.de
}

\author{
Veit Köppen \\ University of Magdeburg \\ Magdeburg, Germany
}

\begin{abstract}
Business processes have to adopt the changes driven by the market to remain competitive. This requires improvement of business processes, which can be carried out by understanding the existing business processes. For this AS-IS business process models are built that represent the actual situation of the enterprise. In this paper, we propose an approach to build an AS-IS process model from employee's descriptions instead of instance executions in information systems. We also present an algorithm that builds an AS-IS model from control flow perspective and we further elaborate it with the help of a case study.
\end{abstract}

\section{Keywords}

Process mining, AS-IS Modeling, task descriptions, model

\section{INTRODUCTION}

Business environments are competitive and changing rapidly, this demands to adopt changes and satisfy customers with quality of products and services. Business processes play a vital role in enterprises and from the last decade have received a considerable attention from researchers $[2,5,18]$. A process consists of activities which can be executed with the support of an information system (partially or completely automated) or can be carried out manually. Enterprises improve their business processes in order to achieve their goals efficiently and in a cost effective way to remain competitive. The objective of business process analysis and improvement is to find out deficiencies in a business process and improve them.

A process model is necessary to improve the business process. Such a model shows the current situation of an enterprise and is called an AS-IS business process model. According to John Wheeler, $10 \%$ to $15 \%$ of cost are spent on

Permission to make digital or hard copies of all or part of this work for personal or classroom use is granted without fee provided that copies are not made or distributed for profit or commercial advantage and that copies bear this notice and the full citation on the first page. To copy otherwise, or republish, to post on servers or to redistribute to lists, requires prior specific permission and/or a fee.

FIT '10, 21-DEC-2010, Islamabad, Pakistan. ACM 978-1-4503-0342-2/10/12 ...\$10.00 technology, whereas $30 \%$ to $40 \%$ are spent on understanding current processes [12]. Understanding existing dependencies between activities is important for business process improvement. Mostly, an AS-IS process model is prepared from instance executions where data is taken from an information system like process mining $[15,8]$ or generated by simulations. The most suitable AS-IS model is the one which consists of activities executed manually as well as activities supported by information systems.

Business process modeling largely depends upon experts and their domain knowledge. Experts get the information about the processes from employees/management and then using their own knowledge to model the business processes. The quality of a process model heavily depends on the experience of the expert which varies from one domain to another. At the end, it's the employees who work for execution of businesses processes and sometimes know more because of their experience of handling a wide range of different cases occur in enterprises.

This paper presents an approach for building an AS-IS model, in which employees are investigated about the working of the system. Afterwards, their descriptions are used as an input to build the AS-IS process model. The proposed approach builds the process model which covers manual and automated activities executed in the system. Furthermore, an algorithm and a case study is discussed to explain the concepts introduced in this paper.

The structure of the paper is as follows: In Section 2, we have discussed business process modeling and its approaches. In Section 3, we further discussed the relation of bottom-up approach for process modeling and AS-IS process model. Section 4, we present the main approach and algorithm, followed by Section 5, where we present a a case study and its benefits. Related work is discussed in Section 6 followed by Section 7 where we provide the conclusion of this paper and mention planned future work.

\section{BUSINESS PROCESS MODELING}

Enterprises divide their overall operations into departments, and further divide into manageable pieces called activities; these activities are executed in a specific order. This spe- 
cific order of activities to generate an output is referred as a business process. The output of a process is used as an input for other processes; therefore, processes collaborate with one another to fulfill the overall objective of an enterprise. Activities can be divided into sub-activities, functions, and tasks which are performed by the users.

A business process model is a representation of activities executed in the system in order to achieve the objective. Being graphical in nature, it is easy for communication with stakeholders, to represent relationships among entities and to understand the overall operations. Business process models are regarded as a core element for process improvement and efficiency [5]. They are also used for modeling the current situation (AS-IS model) running in the enterprises. Once an AS-IS model is available, further analysis for improvement can be made to achieve company's objectives effectively.

Two approaches exist for business processes modeling. One approach is top-down and other one is bottom-up. In the top-down approach, an expert or management propose the model that how business processes should be executed. It starts from an overall process, by considering it as a "black box" and then this "black box" is broken down into more details like activities and tasks until details are specified. In contrast, bottom-up approach starts documenting at a lower level, i.e., how the activities/functions are executed at operational level. After taking this information, tasks/functions are combined into activities, and activities connecting other activities build processes. In this way, the whole business process model is built. Being started from the lower level of details, this approach gives you a detailed insight about the processes and the way they are executed in enterprises.

Top-down and bottom-up approaches are explained in [14] with the context of process improvement from software perspective. In top down approach, a process model is compared with other best practices models, and then identified deficiencies are eliminated from the existing business processes. Whereas in a bottom-up approach, improvement starts from a local domain, and so overall process is improved to achieve the specific goals. Mostly, for business process improvement (or continuous improvement process [6]) bottom-up approach is used, whereas top down is used in business process reengineering [1]; as top down approach do not incorporate organizational problem and unique characteristics [14].

\section{AS-IS MODEL AND BOTTOM-UP}

For business process improvement, an AS-IS model is a prerequisite to understand how processes are executed in the current system. Unlike most object oriented design models, where technical aspects are considered for development, an AS-IS model serves analysts as an important model for understanding and improvement of business processes. Usage of bottom-up approach for an AS-IS modeling ensures that involvement of experts can be kept minimal, therefore, the final AS-IS model would not have an effect of expert's prior knowledge. Bottom-up approach starts from taking the requirements from employees about how processes are executed and then in a stepwise manner the whole business process model is built. In post execution business process modeling, bottom approach is used.
For business process modeling, it is important to represent the involved elements and their relationship with each other. Few elements are briefly discussed as follows, whereas other elements are considered depending on the modeling perspective. For example, modeling the organizational perspective requires information about involved resources like machines and employees.

Involved objects: These are involved in activity processing (like inputs and outputs) and can be physical (like material, goods, and resources) or non-physical (like data and information).

Functions/operations: Resources perform operations on inputs and transform them into outputs. It is important to get answers of different questions to understand the behavior of processes. For example, which functions are performed within activities? What steps have been taken in order to complete the work? What are their execution order like sequence and concurrency?

Conditions and constraints: Different kinds of conditions and constraints occur in processes. These are defined in enterprise policies and applied during execution of activities based on characteristics of involved objects. Conditions play a very important role, as their evaluation decide which activities will be executed and who will execute them? We mention few kinds of conditions below.

Pre-conditions: These are conditions which must be held before the task is executed. First these are validated, then further steps of tasks are executed like required material is available for manufacturing, resource is available to execute the task, and previous activity in sequence is completed.

Post-conditions: These conditions are evaluated at the end of activity (task execution). If these conditions are not met, then it may be required to do the task again or other measures are taken at evaluation.

Other conditions: Different other conditions also exists like logical, event, or time specific, and probabilistic. For example, if there is no reply within one minute then hangup the call, if weight of product is greater than a certain value then particular shipping method, and product manufacturing starts after confirmation of payment.

Constraint: Different constraints are applied on processes based on the characteristics of involved elements. For example, time constraints of organizational resources like employee cannot perform two tasks simultaneously, employees capability (skills), and capacity.

Control structures: Business processes are complex and involve many structures to represent the business processes. In this paper, we deal only with four basic structures, which are called choice, parallelism, sequence, and iteration. Sometimes, due to condition/constraint of the process, a certain task has to be executed from a set of tasks. In this case, choice construct is used to model the process/activities. Similarly, when two or more tasks are independent of one and another then these tasks can be executed independently (or simultaneously), which corresponds to a parallel construct for modeling. When one task is dependent on the result of the previous task, then they are executed one after another and modeled by a sequence construct. When a task has to be repeated more than once, then the itera- 


\begin{tabular}{|c|c|c|c|c|c|c|c|c|c|c|c|}
\hline \multirow{3}{*}{$\begin{array}{l}\text { Steps \& } \\
\text { Alternatives }\end{array}$} & \multicolumn{2}{|c|}{ Objective: } & \multicolumn{3}{|c|}{ Employee id: } & \multicolumn{2}{|c|}{ Process name: } & \multicolumn{3}{|c|}{ Activity name: } & \\
\hline & \multicolumn{3}{|c|}{ Involved entities } & \multicolumn{3}{|c|}{ Conditions } & \multirow[t]{2}{*}{ Functions } & \multirow{2}{*}{$\begin{array}{l}\text { Objects \& } \\
\text { Attributes }\end{array}$} & \multicolumn{3}{|c|}{ Notes } \\
\hline & Input & Output & Others & Pre & Post & Other & & & Next & Previous & Other \\
\hline
\end{tabular}

Table 1: Questionnaire template

tive construct is used. Many other structures are discussed in [16], where author discuss the frequent patterns to model business processes.

\section{APPROACH}

This paper takes the bottom-up approach to build a business process model, i.e., model is designed from the task descriptions of activities as explained by the employees. Therefore, the proposed approach revolves around the employees as they provide the description about execution of business processes. The overall approach is divided into the following phases:

\section{Data collection,}

2. Data extraction (information extraction), and

3. Modeling and analysis.

We are concerned with the control flow perspective of activities in a business process, like which activities are executed, their order of execution (sequence, parallelism), involved conditions, and how they are executed in business processes. Our approach consist of following phases.

\subsection{Data Collection Phase}

We need to know which activities and tasks are performed in process execution to build a process model [5]. For this, employees can report about the execution of activities in a business process. The detailed information can be collected by preparing a questionnaire that would be filled by each employee about their work over activities or they can describe it in text form. In this paper, we use a questionnaire based method. Table 1 shows on questionnaire template to get the employees' description about their tasks. Detail on the following elements is useful for modeling the control flow perspective of business processes.

Tasks information: The objective and description about the tasks like to which activity or process does it belong? For example, the name of task and activity.

Involved objects: Employee describe the elements which are involved in execution of activities as described in previous section. Differentiation between carrier objects and operational objects should be made in the tasks. Carrier objects are those which are used to represent some particular instance, like customer name, order number; whereas the operational objects are those on which operation are performed and changes are made to its values.

Procedure for task execution: Description about task execution like which operations are performed, which conditions are evaluated, what are alternatives procedures and when they should be and relevant details in transforming the input into the output.

Context information: Employees also have an abstract idea of the next and previous activities of a process, therefore, this information is also collected for process modeling.

Other information: Employees also know the dependencies between activities based on certain attributes, therefore, this information should also be collected. Comments from employees on tasks should also be incorporated as different situations occur in operations. However, this information is restricted to their domain only.

\subsection{Extraction Phase}

After collecting the data about tasks from the employees, data which would be helpful for building process model is extracted. The following items can be helpful for process modeling, thus should be extracted from the collected data.

- Activity information (and business process),

- Functions/tasks,

- Involved elements (input, output, and involved objects),

- Conditions (pre and post conditions), and

- Context information (information about next and previous activity).

It is obvious that the information required for process modeling depends on the perspective of a business process being modeled.

\subsection{Modeling and Analysis Phase}

In order to build the process model, relationship between involved elements (functions, input, output, business objects, and their attributes) and activities must be determined. This relationship gives us information about dependencies, conditions, and structural information (like parallelism, choice, and iteration). Therefore, in this phase, control structure of activities is determined. Before describing the algorithm, we require formal definition of a business process and business process model.

Definition 1. A business process $P$ is a set of activities $A$ which are executed in a specific order defined by business rules $R$. These business rules are further specified as involved conditions $C$ and constraints $T$. Organizational resources perform operations on operational objects $O$ and transform them into outputs. Activities itself are composed 


\begin{tabular}{|l|l|}
\hline Sym. & Description \\
\hline$p$ & A process $p$ that belongs to set of processes $P$ \\
\hline$A$ & $A$ is a set of activities which is contained in process $p$ \\
\hline$C$ & $C$ is a set of condition which belongs to a process $p$ \\
\hline$T$ & $T$ is a set of constraint which is related to a process $p$ \\
\hline$\succ$ & Activities are followed by one another (sequence) \\
\hline$\|$ & Activities are parallel \\
\hline$\Longleftrightarrow$ & Similar in description \\
\hline$\phi$ & Empty Set \\
\hline$\cap$ & Intersection \\
\hline
\end{tabular}

Table 2: Symbols and Description

of functions $F$. Activities are connected via edges $E$ in a specific way called control structure.

$$
\begin{gathered}
P:=\{A, E, R\} \\
\text { where } A:=\{F, O\} \text { and } R:=\{C, T, O\}
\end{gathered}
$$

Definition 2. A business process model $M$ is a representation of business process $P$ and its involved elements. Therefore, a business process model $M$ is described as

$$
M:=P\{A, E, R\}
$$

This paper deals with the control flow perspective of a business process, therefore, the following algorithm is proposed to build the business process model from extracted data of a questionnaire. The algorithm is described with the activity level detail, but this algorithm can also be applied at any level of detail like functions and sub processes. Symbols used in specification of algorithm are shown in the Table 2 . Algorithm:

Input: Description about A,C,T

Output:AS-IS process model (control flow perspective)

For each $p$, Read the extracted data for $A, C$, and $T$

- Find control structures

Sequence: Let $a, b$, are two activities, where

$$
\begin{gathered}
a, b \in A, c_{1}, c_{2} \in C, \text { and } t_{1}, t_{2} \in T . \\
\quad \text { if } a\left(c_{1}\right) \cap b\left(c_{2}\right)=\phi \\
\text { AND } a\left(t_{1}\right) \cap b\left(t_{2}\right) \neq \phi
\end{gathered}
$$

then $b \succ a, b$ is followed by $a$, i.e., sequential.

XOR Choice: If

$$
\begin{gathered}
a\left(c_{1}\right) \cap b\left(c_{2}\right) \neq \phi \\
\text { AND } a\left(t_{1}\right) \cap b\left(t_{2}\right) \neq \phi
\end{gathered}
$$

then they have choice construct.

Parallel: If

$$
\begin{gathered}
a\left(c_{1}\right) \cap b\left(c_{2}\right) \neq \phi \\
\text { AND } a\left(t_{1}\right) \cap b\left(t_{2}\right)=\phi
\end{gathered}
$$

then $A \| B$, i.e., they are parallel.

Loop: A loop is a choice construct with a distinction that one of its next activity is already executed.

- Previous activity: Pre-conditions give us information for connecting the activity with previous activity. Let $b\left(c_{2}\right)$ is pre-condition for activity $b$ then this precondition give us information about the previous activity $a$, i.e.,

$$
b\left(c_{2}\right) \Longleftrightarrow a\left(c_{1}\right) \text { similar in description }
$$

Pre-condition of current activity hints on the postcondition of previous activity as well as activity itself.

- Next activity: Similarly, for next activity we already have information about post-condition of current activity $a\left(c_{1}\right)$ then this hints about the next activity $b$.

- When the relation between two functions for modeling is defined then they are marked.

- Steps are repeated for all activities related to a specific process and continued until all processes are modeled.

Once the process is modeled, then experts can be involved to analyze the generated process model for improving the model. Other improvement analyses can be made over that model to increase the efficiency of processes, like what activities and conditions can be added to increase the efficiency or quality of the processes and which sequential activities can be executed in parallel while the cost do not increase. The presented algorithm can work for various levels of abstraction and detail with slight modifications. In the following section, we further discuss the algorithm with the help of a case study.

\section{CASE STUDY AND APPLICATION}

A fictitious case study is carried out with the aim of explaining the algorithm discussed above. The case study is about an enterprise which takes the order from customer, manufacture the products, and deliver them. Major business processes are like "receive orders", "manufacture order", and "ship products". Customer sends the order to a manufacturer for the production with details (like quantity, sizes, and other specifications). After receiving the payment, the order is finalized. Once the order is finalized, the manufacturing process is started (assume stocks of finished products are not maintained). For manufacturing process, a production order with all relevant details specified by the customer is prepared. Based on the production order, material is purchased from the supplier, and actual production of products starts. Manufactured products are shipped to customers and in this way business processes are completed.

For this case study, we take the "manufacture order" process, and specify the sub-process "purchase material". Employees involved in this activity fill out the questionnaire and provides information for modeling as shown in the Table 3. For simplicity in explanation, we have made the assumption that only one supplier exists and will provide the raw material on fixed rates as decided earlier. Similarly, stock of 


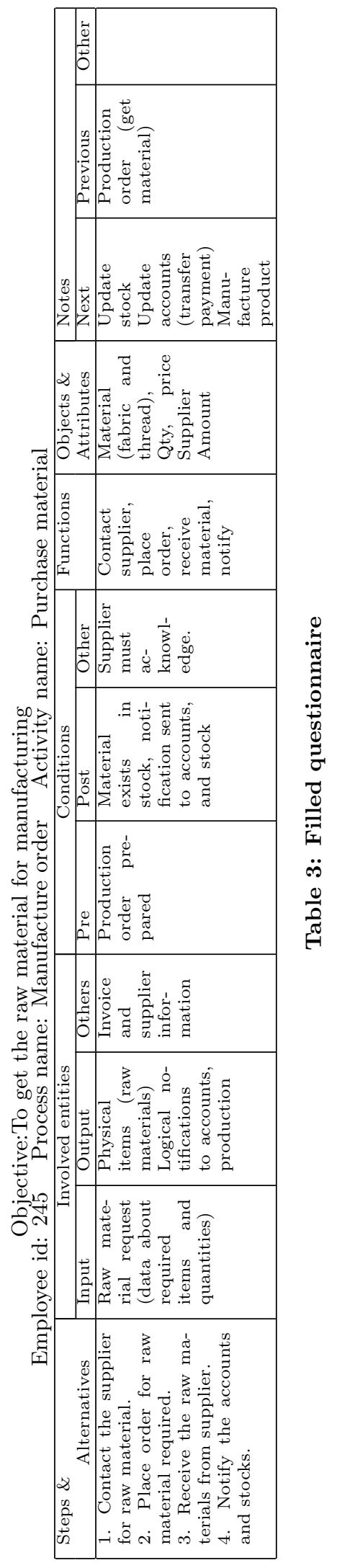

material is not available and the account department transfer the payment to the supplier on notification. Based on the information from Table 3, we apply the algorithm for modeling.

1. First we take a process "manufacture order", activity "purchase material", and its functions. Take its two functions and find the structural relation between them like sequence and parallelism.

2. Sequence construct: For the functions "contact supplier" and "place order", we see that supplier acknowledgment is required before placing the order. Therefore, these activities are sequential in order.

Parallelism construct: For function "notify accounts" and "notify production", there exists only one condition that raw material is received as both only depend on the previous function receive materials. There exists no constraint between two functions, therefore, these two notifications can be carried out independently (in parallel).

Choice and iteration construct: When two functions have same condition but a constraint exists then choice construct is involved. It depends on the constraint and instance which function will be performed. We regard the iterative construct as a choice construct but with an exception that its next activities can be one of it's previously executed activities.

3. Previous activity: Production order is prepared in previous activity (as required raw materials is specified from production order).

4. Next activity: After completion of "receive material", notification is the next activity as post condition of "receive material" is pre-condition of notify.

5. When a relationship between functions/activities is defined, then model them and mark them.

6. Same procedure is repeated for other activities, and processes, therefore, all processes are modeled.

Benefits: This paper presents a technique to build an ASIS model, therefore, applications and benefits are also similar as of an AS-IS model with few more advantages. In comparison to the conventional process mining approaches for building an AS-IS model from information system logs, our presented approach also covers manual activities of the processes and automated activities. Based on this information, analysts can take the decision how to automate them or improve them from best practices as mentioned in $[5$, 11]. Similarly, generated AS-IS model can be used to build a customized solution in order to provide the support for business processes with recent technologies. For this, generated AS-IS model can be compared with the customized solution model and if the new scenario (or enterprise model) matches the customized model, then the same customized product can be recommended or modified accordingly for the enterprise. That saves considerable time and cost in an analysis for devising the solution. Besides other advantages, our presented approach is much useful as compared to the conventional process mining for cases where event logs are 
spread over many IT systems or event logs are not maintained in a structured way.

\section{RELATED WORK}

A business process model is very crucial to get an overall picture of the system and investigated by many researchers in various directions $[17,5,18]$. Different modeling perspectives like object, activity, and control are discussed in [17, 4, $5,13]$. UML diagrams are transformed from one to another like from sequence diagrams to state chart as well as from source code as discussed in $[10,19]$ with the focus towards iterative software development. However, with these transformations, correctness has to be verified as described in [3]. In [9], authors present an approach to improve the existing AS-IS business processes by process merging techniques with reference models and best practices.

The work related to the proposed approach is discussed in [3], where the author involves domain experts to define the instances and then presented an approach to build a process model using Petri nets formalism. In [7], authors present an approach to construct two models from task descriptions to support processes with technologies. One model helps in requirement elicitation and validation by providing prototype like representation and simulation, whereas another model helps to implement IT system according to the requirements to fulfill the gap between task descriptions and actual IT support.

\section{CONCLUSION AND FUTURE WORK}

In this paper, we presented an algorithm with a case study which can be used to build an AS-IS process model with the help of a questionnaire. The presented work makes a foundation for business process improvement and self adaptive customization. In the future, research on following aspects will be carried out: 1) evaluation and improvement of proposed approach with other control flow patterns; 2) semantic based approach for finding relationship between activities from descriptions; 3) extending it for other modeling perspectives; 4) self adaptive customization.

\section{Acknowledgment}

Azeem Lodhi is supported by a grant from the federal state of Saxony-Anhalt in Germany. This work is supported by the German Ministry of Education and Science (BMBF), within the VierforES ${ }^{1}$ project No. 01IM08003C. The presented work is part of the ViERforES project.

\section{REFERENCES}

[1] J. Clemmer. Process reengineering and process improvement: not an either /or choice. CMA Magazine, 68:36-39, 1994.

[2] R. Davis and E. Brabänder. ARIS Design Platform: Getting Started with BPM. Springer, 1 edition, June 2007.

[3] J. Desel. From human knowledge to process models. In R. Kaschek, C. Kop, C. Steinberger, and G. Fliedl, editors, UNISCON, volume 5 of Lecture Notes in Business Information Processing, pages 84-95. Springer, 2008.

\footnotetext{
${ }^{1}$ www.vierfores.de
}

[4] M. Dowson. Iteration in the software process; review of the 3rd international software process workshop. In Proceedings of the 9th international conference on Software Engineering, pages 36-41, Los Alamitos, CA, USA, 1987. IEEE Computer Society Press.

[5] M. Dumas, W. van der Aalst, and A. ter Hofstede. Process Aware Information Systems: Bridging People and Software Through Process Technology. Wiley \& Sons, 2005.

[6] M. Imai. Kaizen: The Key To Japan's Competitive Success. McGraw-Hill, 1986.

[7] J. B. Jorgensen, K. B. Lassen, and W. van der Aalst. From task descriptions via colored petri nets towards an implementation of a new electronic patient record workflow system. Int. J. Softw. Tools Technol. Transf., 10(1):15-28, 2007.

[8] A. Khan, A. Lodhi, V. Köppen, G. Kassem, and G. Saake. Applying process mining in SOA environments. In A. Dan, F. Gittler, and F. Toumani, editors, Service-Oriented Computing ICSOC Service Wave 2009 Workshops, volume 6275 of Lecture Notes in Computer Science, pages 293-302. Springer, 2010.

[9] J. M. Küster, J. Koehler, and K. Ryndina. Improving business process models with reference models in business-driven development. In J. Eder and S. Dustdar, editors, Business Process Management Workshops, volume 4103 of Lecture Notes in Computer Science, pages 35-44. Springer, 2006.

[10] T. Maier and A. Zündorf. The fujaba statechart synthesis approach. In 2nd International Workshop on Scenarios and State Machines: Models, Algorithms, and Tools, Oregon, Portland, USA, May 2003. Wiley-VCH.

[11] S. L. Mansar and H. Reijers. Best practices in business process redesign: Survey results amongst dutch and uk consultants. In Proceedings of the 2004 Research Conference on Innovations in Information Technology (IIT, pages 413-420, Dubai, 2004. UAE University.

[12] E. Monk and B. Wagner. Concepts in Enterprise Resource Planning. Course Technology, 2 edition, March 2005.

[13] A.-W. Scheer. ARIS-Business Process Frameworks. Springer-Verlag, New York, Inc., 2 edition, 1998.

[14] M. Thomas and F. McGarry. Top-down vs. bottom-up process improvement. IEEE Software, 11(4):12-13, July 1994.

[15] W. van der Aalst and A.J.M.M.Weijters. Process mining: A research agenda. Computers in Industry, 53:231-244, 2004.

[16] W. van der Aalst, A. ter Hofstede, B. Kiepuszewski, and A. Barros. Workflow patterns. Distrib. Parallel Databases, 14(1):5-51, 2003.

[17] W. van der Aalst and K. van Hee. Workflow Management: Models, Methods, and Systems. MIT Press, Cambridge, Massachusets, UK, 2002.

[18] M. Weske. Business Process Management: Concepts, Languages, Architectures. Springer Verlag, 1 edition, November 2007.

[19] J. Whittle and J. Schumann. Generating statechart designs from scenarios. In Proceedings of the 22nd International Conference on Software Engineering, pages 314-323, New York, NY, USA, 2000. ACM. 